\title{
ARTICLE \\ Metoprolol prevents neuronal dendrite remodeling in a canine model of chronic obstructive sleep apnea
}

\author{
Lin Yang ${ }^{1}$, Jing Zhao ${ }^{2}$, Yang Qu ${ }^{1}$, Qiang Sun ${ }^{1}$, Ting-ting $\mathrm{Li}^{1}$, Mei-ling Yan ${ }^{1}$, Ming-jing Duan ${ }^{1}$, Ke-xin Li ${ }^{1}$, Yan-ru Wang ${ }^{1}$, Si-yu Huang ${ }^{1}$,
} Shuai Zhang ${ }^{1}$, Yue $\mathrm{Li}^{2}$ and Jing $\mathrm{Ai}^{1}$

\begin{abstract}
Obstructive sleep apnea (OSA) is closely associated with central nervous system diseases and could lead to autonomic nerve dysfunction, which is often seen in neurodegenerative diseases. Previous studies have shown that metoprolol prevents several chronic OSA-induced cardiovascular diseases through inhibiting autonomic nerve hyperactivity. It remains unclear whether chronic OSA can lead to dendritic remodeling in the brain, and whether metoprolol affects the dendritic remodeling. In this study we investigated the effect of metoprolol on dendrite morphology in a canine model of chronic OSA, which was established in beagles through clamping and reopening the endotracheal tube for $4 \mathrm{~h}$ every other day for 12 weeks. OSA beagles were administered metoprolol $\left(5 \mathrm{mg} \cdot \mathrm{kg}^{-1} \cdot \mathrm{d}^{-1}\right)$. The dendritic number, length, crossings and spine density of neurons in hippocampi and prefrontal cortices were assessed by Golgi staining. And the protein levels of hypoxia-inducible factor-1a (HIF-1a) and brain-derived neurotrophic factor (BDNF) were measured by Western blotting. We showed that chronic OSA successfully induced significant brain hypoxia evidenced by increased HIF-1a levels in CA1 region and dentate gyrus of hippocampi, as well as in prefrontal cortex. Furthermore, OSA led to markedly decreased dendrite number, length and intersections, spine loss as well as reduced BDNF levels. Administration of metoprolol effectively prevented the dendritic remodeling and spine loss induced by chronic OSA. In addition, administration of metoprolol reversed the decreased BDNF, which might be associated with the metoprolol-induced neuronal protection. In conclusion, metoprolol protects against neuronal dendritic remodeling in hippocampi and prefrontal cortices induced by chronic OSA in canine.
\end{abstract}

Keywords: metoprolol; chronic obstructive sleep apnea; canine model; hippocampus; prefrontal cortex; neuronal dendritic remodeling; HIF-1a; BDNF

Acta Pharmacologica Sinica (2020) 41:620-628; https://doi.org/10.1038/s41401-019-0323-8

\section{INTRODUCTION}

Obstructive sleep apnea (OSA) is frequently seen in sleepdisordered breathing, which is found in $10 \%$ of people aged 30-70 years. OSA is characterized by repeated partial or complete obstruction of the upper airway that leads to arousal during sleep due to reduced airflow [1-3]. A large number of studies demonstrated that OSA is closely related to cardiovascular diseases (CVDs), such as hypertension [4], arrhythmia [5], heart failure (HF) [6], and coronary artery disease [7]. At the same time, abundant evidence shows that OSA patients are more likely to develop cerebrovascular disease or dementia $[8,9]$. It has been reported in a clinical study that OSA patients with subjective cognitive impairment display worse memory, attention and intelligence performance than patients with subjective cognitive impairment alone [10]. The mean survival for OSA patients with amyotrophic lateral sclerosis (ALS) was dramatically shorter than that for patients without OSA [11]. In addition, depression is also a common comorbidity of OSA [12]. However, how OSA induces these pathological processes is largely unknown.

Dendritic degeneration and synapse loss are the pathological hallmarks contributing to Huntington's disease (HD), Alzheimer's disease (AD), ALS, and depression [13-16]. Typically, intermittent hypoxia $(\mathrm{IH})$ simulating repetitive hypoxia and reoxygenation is an approach to mimic the features of OSA [17]. A previous study reported that the exposure of 10-day-old rats to $\mathrm{IH}$ chambers for 2 weeks resulted in decreased neuron dendritic branching in the frontal cortex during adulthood [18]. Exposure of adult mice to $\mathrm{IH}$ also induced memory decline, accompanied by reduced spine number in the CA1 [19]. These studies suggested that IH exposure at the developmental stage or in adulthood resulted in long-term neuronal damage and neurobehavioral deficits. Apart from the rodent model of OSA, dogs are also extensively utilized in this field [20]. Repeated airway occlusion in canines is a successful approach to examine the mechanism underlying OSA [21], and provides a reliable and modifiable model of OSA, thereby allowing simultaneous neural manipulation and hemodynamic measurements [20]. To date, it is still unclear whether chronic OSA causes dendritic remodeling in canine models.

It has been reported that sleep disorder is associated with the deregulation of the autonomic nervous system [22]. Indeed, our previous study demonstrated that the canine model of chronic OSA displayed autonomic nerve hyperinnervation [23]. Autonomic

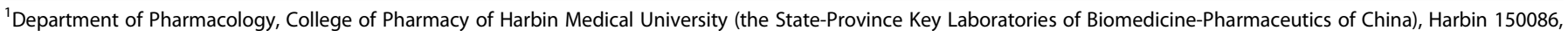
China and ${ }^{2}$ Department of Cardiology, the First Affiliated Hospital, Key Laboratory of Cardiac Diseases and Heart Failure, Harbin Medical University, Harbin 150086, China Correspondence: Jing Ai (azhrbmu@126.com) or Yue Li (ly99ly@vip.163.com)

Received: 1 August 2019 Accepted: 26 October 2019

Published online: 20 December 2019 
nerve dysfunction is also a cardinal feature of $A D$, Parkinson's disease (PD) and depression [24-26]. Nevertheless, it remains unclear whether the autonomic nervous system is involved in OSA-associated CNS disorders.

Metoprolol, a selective $\beta 1$-adrenergic receptor antagonist, prevented chronic OSA-induced atrial fibrillation by inhibiting autonomic nerve hyperactivity [27], and it inhibited cardiac apoptosis as well as interstitial fibrosis in a canine model of chronic OSA [28]. Furthermore, metoprolol prevented profibrotic remodeling of epicardial adipose tissues in a canine model of chronic OSA [29]. In addition to the multiple therapeutic functions of metoprolol in OSA-induced cardiovascular lesions, metoprolol also works in CNS disorders [30]. Specifically, a transient reduction in PD symptoms (rigidity) is recorded when suppressing the subthalamic nucleus spiking activity in PD patients after metoprolol application [30]. Although the underlying mechanism of action of metoprolol remains unclear, the potential use of metoprolol in PD is supported. To the best of our knowledge, there is no report about the effects of metoprolol on neuronal dendritic remodeling in any OSA animal model. Therefore, this study aimed to address this question by evaluating the role of metoprolol in dendritic morphological changes in the chronic OSA canine model. We first found that metoprolol effectively ameliorated dendritic degeneration and spine loss in chronic OSA and that BDNF might be involved in this process.

\section{MATERIALS AND METHODS}

Animals and the chronic OSA protocol

All experiments in this study were approved by the ethics committees of Harbin Medical University, and were performed in compliance with the Guide for the Care and Use of Laboratory Animals published by the US National Institutes of Health (8th edition, 2011). Twelve male beagles (weight $15-20 \mathrm{~kg}$, from the
Experimental Animal Center of the First Affiliated Hospital of Harbin Medical University) were housed in cages under standard laboratory conditions. Beagles were anesthetized with intravenous ketamine $\left(5.3 \mathrm{mg} \cdot \mathrm{kg}^{-1}\right)$, diazepam $\left(0.25 \mathrm{mg} \cdot \mathrm{kg}^{-1}\right)$, and xylazine $\left(1 \mathrm{mg} \cdot \mathrm{kg}^{-1}\right)$. The adequacy of anesthesia was monitored in light of the disappearance of the corneal reflex and jaw tone. The sham group underwent anesthesia and tracheal intubation only. For OSA beagles, under anesthesia, the tubes were clamped to elicit apnea at the end of the exhalation after the tracheal tubes were intubated into the trachea. The protocol for chronic OSA was performed according to previous studies [23, 28, 29]. Briefly, chronic OSA stimulation was maintained for 12 weeks. In the first week, the duration of $1 \mathrm{~min}$ was set as the trachea blockage time frame, and 9 min was set as the ventilation time frame. In the next 3 weeks, the duration of ventilation was $1 \mathrm{~min}$ shorter progressively than the previous week. In the following 8 weeks, the duration of trachea blockage was $1 \mathrm{~min}$, and trachea ventilation was $5 \mathrm{~min}$. During the 12 weeks, OSA stimulation was performed once every 2 days and was maintained for $4 \mathrm{~h}$ each day. All beagles were kept under anesthesia for $4 \mathrm{~h}$ during the chronic OSA periods and were drug-free for the remainder of the time. The details of the OSA stimulation protocol are shown in Fig. 1a. A total of 12 animals were randomly divided into three groups (four beagles for each group): the sham group, the OSA group and the OSA treated with metoprolol group (AstraZeneca, ZOK 50, $5 \mathrm{mg}$. $\left.\mathrm{kg}^{-1} \cdot \mathrm{d}^{-1}\right)$. The selection of the dosage of metoprolol was based on a previous study and the introduction of dose conversion between humans and beagles [27, 29, 31].

Negative intra-airway pressure measurement

To record the negative intra-airway pressure, animals were anesthetized. First, the baseline intra-airway pressure was monitored in at least two complete respiratory cycles under quiet, supine respiration status. We next recorded the negative

a

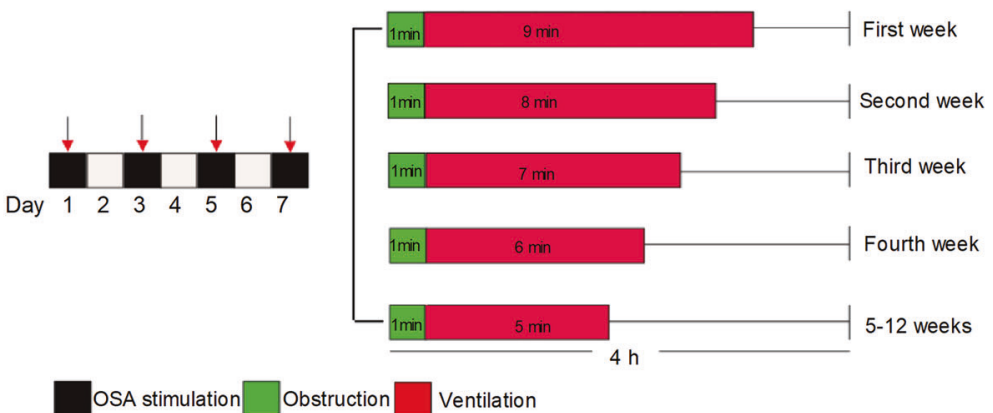

b

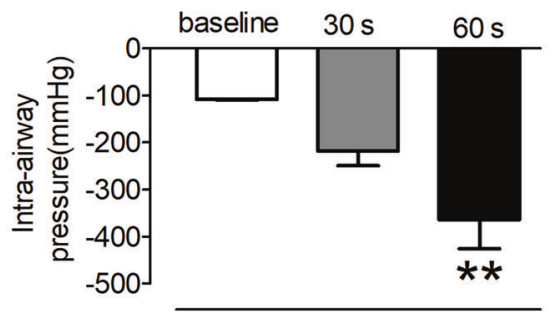

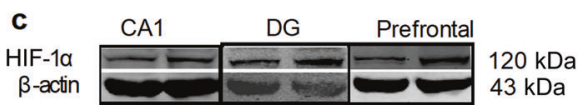

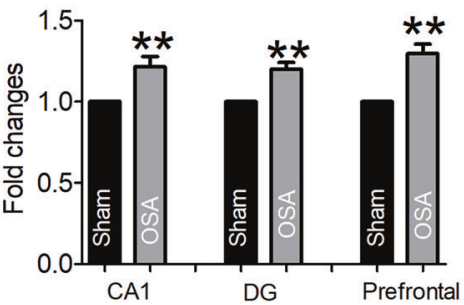

Fig. 1 Construction of the OSA canine model. a Schematic diagram of the chronic OSA beagle model. At the first week, the apnea AHI was set as 6 . The duration of trachea blockage was $1 \mathrm{~min}$, and ventilation was $9 \mathrm{~min}$. In the next 3 weeks, the ventilation duration was progressively 1 min shorter than the previous week. In the last 8 weeks, the duration of tracheal ventilation was 5 min, and the AHI was 10 . The ventilation and blockage were exchanged for $4 \mathrm{~h}$ every other day in the whole process. b Negative intra-airway pressure at baseline and apnea for 30 and 60 s. ${ }^{* *} P<0.01$ vs. baseline, $n=4$ for each group, and each value represents the mean \pm SEM. $c$ HIF- $1 \alpha$ expression level in the hippocampal CA1 and DG as well as in the prefrontal cortex (for CA1: $n=7$; DG: $n=5$; prefrontal cortex: $n=5$, with four beagles per group). ${ }^{* *} P<0.01$ vs. sham control group, values are expressed as the mean \pm SEM. 
intra-airway pressures by closing the exit valve for either 30 or $60 \mathrm{~s}$ at the normal end-tidal expiration.

\section{Western blotting}

Brain tissue samples were harvested and stored at $-80^{\circ} \mathrm{C}$. Total protein samples for Western blot analysis were extracted from the CA1 and DG of the hippocampus or superficial prefrontal cortex of the beagle, and the detailed preparation protocol was as follows. Frozen brain tissues were lysed in a solution containing $40 \%$ SDS, $60 \%$ RIPA, and $1 \%$ protease inhibitor. The homogenate was then centrifuged at $16700 \times g$ at $4{ }^{\circ} \mathrm{C}$ for $30 \mathrm{~min}$, and the supernatants (containing cytosolic and membrane fractions) were collected. The concentration of proteins was detected spectrophotometrically using a BCA kit (Universal Microplate Spectrometer; Bio-Tek Instruments, Winooski, VT, USA). Protein samples were fractionated by $10 \%$ SDS-PAGE gels and then transferred onto a nitrocellulose membrane. Anti-BDNF (1:1000, ab108319, Abcam, MA, USA) and anti-HIF-1a (1:1000, 12-2180, Assay Biotechnology, CA) were used as primary antibodies. $\beta$-Actin (1:1000, AT-09, ZSGB$\mathrm{BIO}$, China) was selected as an internal control. Blots were detected using an Odyssey Infrared Imaging System (Licor, Lincoln, NE, USA) and were quantified with Odyssey v1.2 software by measuring the protein intensity (area $\times$ optical density) in each group. The final results were expressed as fold changes compared with the control values.

\section{Golgi staining and Sholl analysis}

The fresh brains were removed and immediately processed using the FD Rapid Golgi Stain Kit (FD Neurotechnologies, Columbia, SC, USA) according to the manufacturer's protocol. The different regions of brain tissues were immersed in the 1:1 mixture of solutions $A$ and $B$ and stored at room temperature avoiding light for 1 day, followed by replacement with fresh solution and storage for another 2 weeks. Under the same conditions, the brain tissues were then transferred into solution $C$ for 1 day, followed by incubation for another 3 days in fresh solution C. After this process, the brain tissues were cut into $120 \mu \mathrm{m}$ slices, rinsed two times in Milli-Q water for $4 \mathrm{~min}$, and then placed into a mixed solution composed of solution $D$, solution $E$ and Milli-Q water at a ratio of $1: 1: 2$ for $10 \mathrm{~min}$. The brain slices were rinsed twice with Milli-Q water for $4 \mathrm{~min}$. Next, the brain slices were placed in a series of $50 \%, 75 \%$ and $95 \%$ ethanol for $4 \mathrm{~min}$ each and rinsed four times with absolute ethanol for $4 \mathrm{~min}$ for dehydration. Finally, slices were placed in xylene for $4 \mathrm{~min}$ and washed three times for clearing and coverslipping with Permount. For image collection, bright-field microscopy was used with an $\times 10$ or $\times 63$ objectives using a Zeiss Axio Scope A1 microscope. Neurons in the CA1 and DG regions of the hippocampi and in layer II/III of the prefrontal cortices in each group were randomly selected from four brains, and 3 slices/brain were quantified. For dendritic spine analysis, dendrites from 2 cells/slice randomly observed in 3 slices/brain were included in each group. Dendritic spines were counted using the following criteria: the primary basal dendrites were defined at the middle between the soma and distal termination, and the secondary apical dendrites were sampled from the midpieces of dendritic ramifications from the primary dendrites. For each selected sample, the analyzed branch length was at least $10 \mu \mathrm{m}$. The spine number was counted within the corresponding segment dendritic branch. The dendritic spine density was evaluated as the ratio of the spine number/the length of the dendritic branch. The selected neurons were traced and reconstructed after switching to 8 bit using ImagePro Plus software. The dendritic complexity was determined by Sholl analysis, in which, with the neuronal body as the origin, concentric circles $10 \mu \mathrm{m}$ apart were drawn until reaching the end of the farthest dendrite and the dendrite crossings on every circle were then recorded (Fig. $2 \mathrm{~h}$ ).
Statistical analysis

Data are described as the mean \pm standard error of the mean (SEM). Each data set was analyzed for the equality of the variance. The independent sample test was calculated using the Levene variance equality test. If $P>0.05$, an independent Student's $t$ test was used for the comparison between two groups; if $P<0.05$, the Kruskal-Wallis rank sum test was performed. Statistical analysis was performed using one-way ANOVA with post hoc tests by using Fisher's test of least significant difference (LSD-t) for comparisons of more than two groups. For Sholl analysis, the effects of group and distance from the soma on dendritic complexity were analyzed by using the general linear model (GLM), with repeated measures for distance from the soma, followed by LSD-t. $P<0.05$ was considered statistically significant. SPSS22.0 was used for all statistical analyses, and graphs were generated using GraphPad Prism 5.0 software (La Jolla, CA, USA).

\section{RESULTS}

Development of the chronic OSA model in canines

To establish the chronic OSA model in canines, the tracheas of beagles were clamped using a tracheal tube to alternately obstruct and ventilate the airway for $4 \mathrm{~h}$ /day maintained for 12 weeks (Fig. 1a). Successful development of the OSA model in canines was evaluated by successfully eliciting the gradually increased negative intra-airway pressure. As illustrated in Fig. 1b, compared with baseline intra-airway pressure in the sham group $(-107.75 \pm 2.25 \mathrm{mmHg})$, the intra-airway pressure progressively decreased to $-218.75 \pm 30.16 \mathrm{mmHg}$ and $-363.75 \pm 61.83 \mathrm{mmHg}$ after valve occlusion for 30 and $60 \mathrm{~s}$, respectively. To double-check the establishment of the OSA canine model, we detected the expression of HIF-1a, which is an oxygen-sensitive heterodimeric transcription factor considered a common indicator of hypoxia [32]. As predicted, the expression of HIF-1a was increased in the hippocampal CA1 and DG as well as in the prefrontal cortices of the chronic OSA group in comparison to the sham group (Fig. 1c). These data suggest that the OSA model in canines had been successfully established.

Metoprolol rescues dendritic remodeling of the hippocampal CA1 pyramidal cells of chronic OSA beagles

Dendrites, emerging from the soma of neurons, play a very important role in the central nervous system [33]. To observe the effects of chronic OSA on neuronal dendrite morphology in the hippocampal CA1 of beagles, we performed Golgi staining as previously reported $[34,35]$. We found that chronic OSA induced a dramatic reduction in the length and number of total, secondary and tertiary dendrites from pyramidal neurons in the hippocampal CA1 of OSA beagles without changes in primary dendrites (Fig. $2 a-c)$. Although the function of both apical and basal dendrites is to ensure that pyramidal cells could receive synaptic inputs from afferent sources and integrate into circuits to spread neuronal communications, they accept information from different afferent sources because they develop from different sides of the cone-shaped soma of pyramidal cells [36]. This means that the morphological remodeling of these two regions would induce different pathophysiological changes. Therefore, we evaluated apical and basal dendrites separately. We found that, compared with the sham group, chronic OSA dramatically decreased the length and number of apical dendrites in total and tertiary dendrites without changing primary and secondary dendrites (Fig. 2d, f). For basal dendrites, chronic OSA reduced the length and number of not only total and tertiary dendrites but also secondary dendrites (Fig. 2e, g). A previous study reported that obstructed respiration increased the susceptibility to atrial fibrillation by autonomic nerve activation [37]. Blocking autonomic nerve hyperinnervation by metoprolol prevented OSA-induced hypertension [38]. Surprisingly, we found that metoprolol 


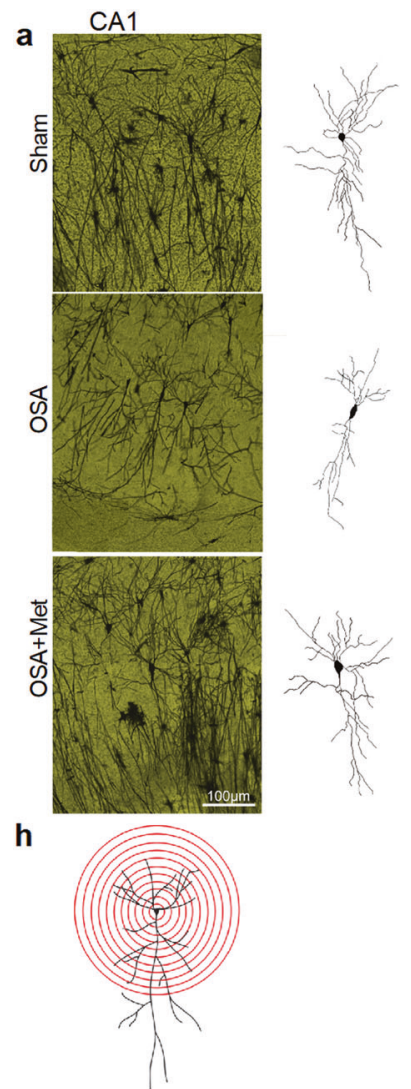

b

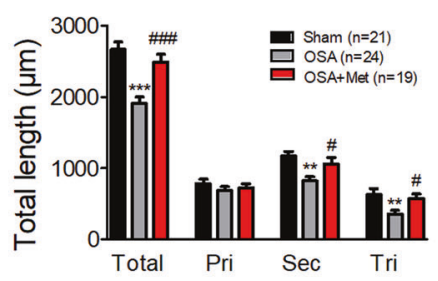

e
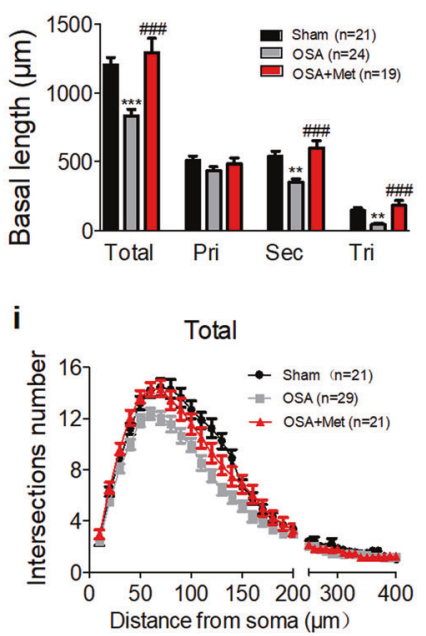

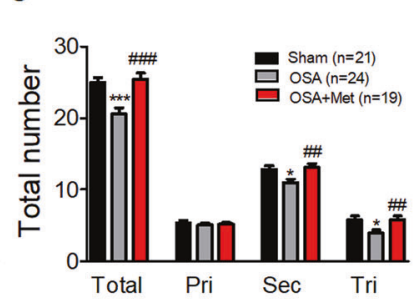

f
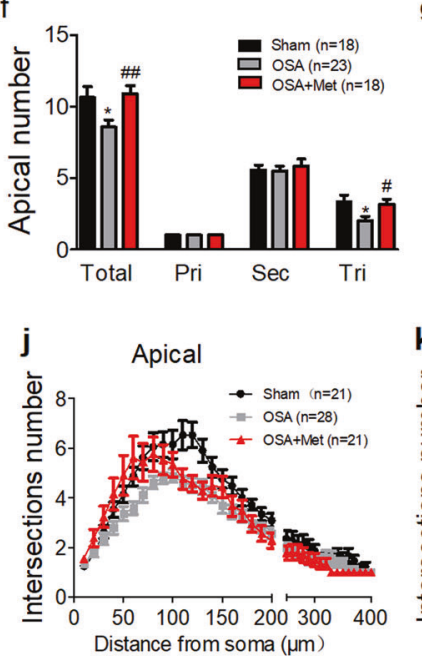

d
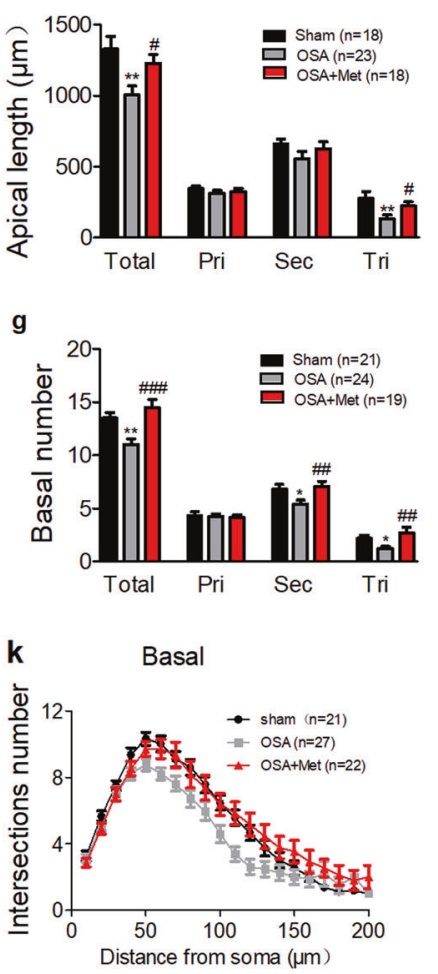

Fig. 2 Metoprolol rescues the degeneration of pyramidal neurons in the hippocampal CA1 of the OSA canine model. a Representative photomicrograph (left) and tracing image (right) of pyramidal neurons in the hippocampal CA1 from the sham control group (upper), OSA group (middle) and OSA treated with metoprolol group (below), scale bar $=100 \mu \mathrm{m}$. $\mathbf{b}-\mathbf{g}$ Quantification of total dendrite length (b), total dendrite number (c), apical dendrite length (d), basal dendrite length (e), apical dendrite number (f), and basal dendrite number (g) in hippocampal pyramidal neurons. $\mathbf{h}$ Schematic experimental diagram of Sholl analysis. $\mathbf{i}-\mathbf{k}$ Sholl analysis of the intersection number of total dendrites $(10-200 \mu \mathrm{m})(\mathbf{i})$, apical dendrites $(10-200 \mu \mathrm{m})(\mathbf{j})$ and basal dendrites $(10-120 \mu \mathrm{m})(\mathbf{k}) .{ }^{*} P<0.05,{ }^{*} P<0.01,{ }^{* * *} P<0.001 \mathrm{vs}$. sham control group, ${ }^{\#} P<0.05,{ }^{\# \#} P<0.01,{ }^{\# \# \# P}<0.001$ vs. OSA group, values are expressed as the mean \pm SEM.

dramatically reversed all the dendritic remodeling induced by chronic OSA (Fig. $2 \mathrm{~b}-\mathrm{g}$ ). Neuronal dendritic complexity reflects the function of the neural network. Next, we analyzed the dendritic complexity using Sholl analysis (Fig. 2h). The intersection numbers of overall dendrites (Fig. 2i), apical dendrites (Fig. 2j) and basal dendrites (Fig. 2k) were all significantly reduced in chronic OSA beagles. Identically, metoprolol markedly reversed all the decreased dendritic complexity (Fig. 2i-k). These findings suggested that metoprolol could rescue the neuronal dendritic regression induced by chronic OSA in hippocampal CA1 pyramidal neurons.

Metoprolol reverses the dendritic degeneration of prefrontal cortical pyramidal neurons induced by OSA

In addition to the hippocampus, the prefrontal cortex is an important brain area encoding learning and memory information. Next, we quantified the dendrite number and length by tracing the pyramidal neuronal dendrites in layer II/III of the prefrontal cortex. Representative graphs and tracing images are shown in Fig. 3a. Compared with the control group, the overall number and length of total, secondary and tertiary dendrites were markedly reduced in chronic OSA beagles without changes in primary dendrites, and metoprolol treatment powerfully rescued the dendritic regression (Fig. 3b, c). Similar results were observed in apical dendrites (Fig. 3d, f) and basal dendrites (Fig. 3e, g). Furthermore, Sholl analysis results indicated that metoprolol attenuated the decreased dendritic complexity of total (Fig. 3h), apical (Fig. 3i) and basal dendrites (Fig. 3j) in chronic OSA beagles. These results indicated that metoprolol could also prevent chronic OSA-induced pyramidal neuronal dendritic degeneration in the prefrontal cortex.

Metoprolol treatment ameliorates the degeneration of granular cells in the hippocampal DG and nonpyramidal cells in the prefrontal cortices of chronic OSA beagles

Granular cells are the principal neurons in the hippocampal DG receiving the primary input from the cortex via the perforant path, and their axons form mossy fibers, which play an important role in spatial memory formation [39]. To investigate whether OSA leads to dentate granular cell degeneration, we also analyzed the morphological changes of granular neurons in the hippocampal DG by Golgi staining (Fig. 4a). We found that chronic OSA decreased the length and number of granular dendrites in total, secondary and tertiary dendrites without changing primary dendrites, and metoprolol administration could rescue all these changes (Fig. 4b, c). Histologically, the cerebral cortex is generally divided into six layers, and the neurons mainly distributed across all the layers are pyramidal and nonpyramidal neurons. The excitatory pyramidal neurons and the inhibitory nonpyramidal interneurons contribute to the microcircuits of the cortex [40]. Prefrontal layer II/III neurons are closely associated with stress and cognition, and most studies focused on examining morphological changes in these two layers [41-44]. Therefore, we observed the morphological changes of nonpyramidal neurons in layer II/III of 

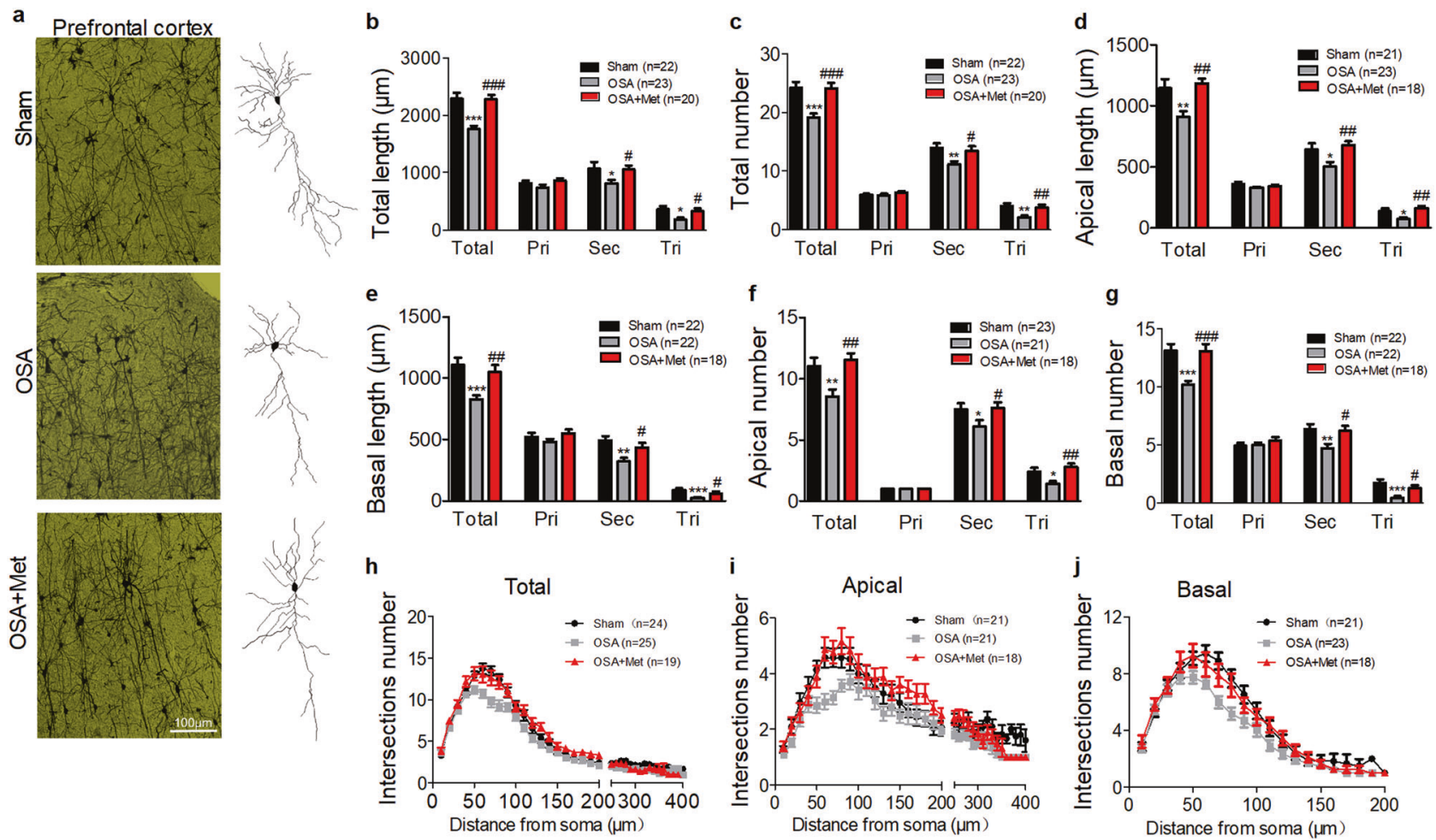

Fig. 3 Metoprolol reverses the degeneration of prefrontal pyramidal neurons in the OSA canine model. a Representative photomicrograph (left) and tracing image (right) of pyramidal neurons in prefrontal cortices from the sham control group (top), OSA group (middle) and OSA with metoprolol treatment group (bottom), scale bar $=100 \mu \mathrm{m}$. $\mathbf{b}-\mathbf{g}$ Quantification of prefrontal pyramidal overall dendrite length (b), overall dendrite number (c), apical dendrite length (d), basal dendrite length (e), apical dendrite number (f), and basal dendrite number $(\mathbf{g}) . \mathbf{h}-\mathbf{j}$ Sholl analysis of the intersection number of total dendrites $(10-200 \mu \mathrm{m})(\mathbf{h})$, apical dendrites $(10-200 \mu \mathrm{m})(\mathbf{i})$ and basal dendrites $(10-120 \mu \mathrm{m})(\mathrm{j}) .{ }^{*} P<0.05,{ }^{* *} P<0.01,{ }^{* *} P<0.001$ vs. sham control group, ${ }^{\#} P<0.05,{ }^{\# \#} P<0.01$, ${ }^{\# \#} P<0.001$ vs. OSA group, values are expressed as the mean \pm SEM.

the prefrontal cortex. The length and number of prefrontal nonpyramidal dendrites in all dendrites were significantly decreased in the chronic OSA beagles, and this was dramatically prevented by metoprolol (Fig. $4 d-f$ ). Taken together, metoprolol treatment could effectively rescue granular dendrite regression in the hippocampal DG and inhibit nonpyramidal degeneration in the prefrontal cortex of the OSA canine model.

Metoprolol prevents dendritic spine loss in the OSA canine model Dendritic spines are dynamic structures, and their addition and elimination are interpreted as excitatory synapse gain and loss, which is considered excitatory circuit remodeling [45]. Next, we detected the dendritic spine density in the hippocampi and prefrontal cortices from the sham group, OSA group and OSA treated with metoprolol group (Fig. 5). The results demonstrated that chronic OSA dramatically decreased the spine density of the apical and basal dendrites in both hippocampi (Fig. 5e, f) and prefrontal cortices (Fig. $5 \mathrm{~g}, \mathrm{~h}$ ) in comparison to the sham group. Surprisingly, metoprolol treatment significantly reversed the spine loss induced by chronic OSA (Fig. 5). These data demonstrated that metoprolol could effectively alleviate spine loss in the hippocampus and prefrontal cortex in chronic OSA.

Metoprolol alleviates the dendritic remodeling induced by chronic OSA via the upregulation of BDNF

BDNF supports a variety of functions, including the regulation of neuronal morphology and synaptic plasticity [46]. A previous study indicated that BDNF-mutant mice displayed a significant reduction in dendritic development, synaptic formation and maturation in postnatal-born granule neurons [47]. Decreased levels of BDNF have been reported in neurodegenerative diseases, including $A D, P D$, and $H D$, which are closely associated with neuron degeneration $[48,49]$. We hence evaluated the expression of BDNF protein as collateral evidence to evaluate OSA-induced dendritic remodeling. As illustrated in Fig. 6, BDNF was significantly decreased in the hippocampal CA1 and DG regions as well as in the prefrontal cortex of the chronic OSA group compared with those in the control group. These results provide further evidence that OSA can induce dendritic spine degeneration. Strikingly, metoprolol treatment dramatically rescued the decrease in BDNF expression in the brain of OSA beagles (Fig. 6). These findings suggest that the ameliorative effects of metoprolol on OSA-induced dendritic degeneration involve the upregulation of BDNF expression.

\section{DISCUSSION}

OSA is a common comorbidity in CVDs [4-7] and CNS diseases $[9,11-13]$. Dendrite degeneration and dendritic spine loss are considered hallmarks of the pathology of many CNS diseases, such as HD, AD, ALS and depressive disorder [13-16]. However, whether a canine model of chronic OSA can lead to neuronal dendritic degeneration remains largely unclear. In the present study, we first reported that chronic OSA (12 weeks) beagles displayed marked dendritic remodeling and spine loss in their hippocampi and prefrontal cortices. Surprisingly, metoprolol could effectively prevent the dendrite regression and spine loss induced by chronic OSA.

Some experimental methods are used to establish animal models of IH to simulate the repetitive hypoxic character of OSA. For example, a common method was used to establish rodent models of OSA to keep rodents in a special chamber where the 
a

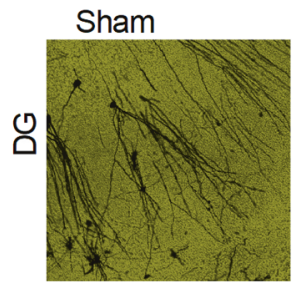

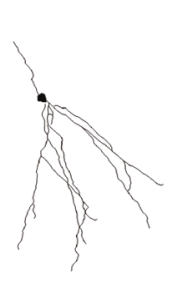
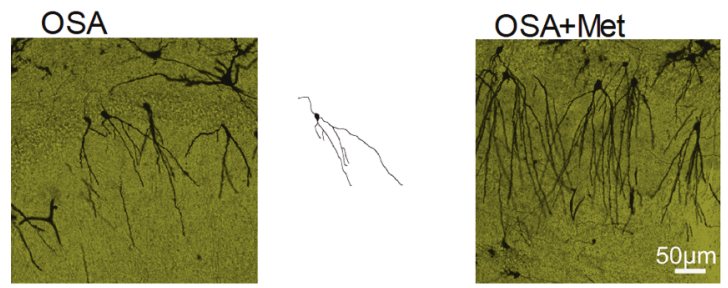

C
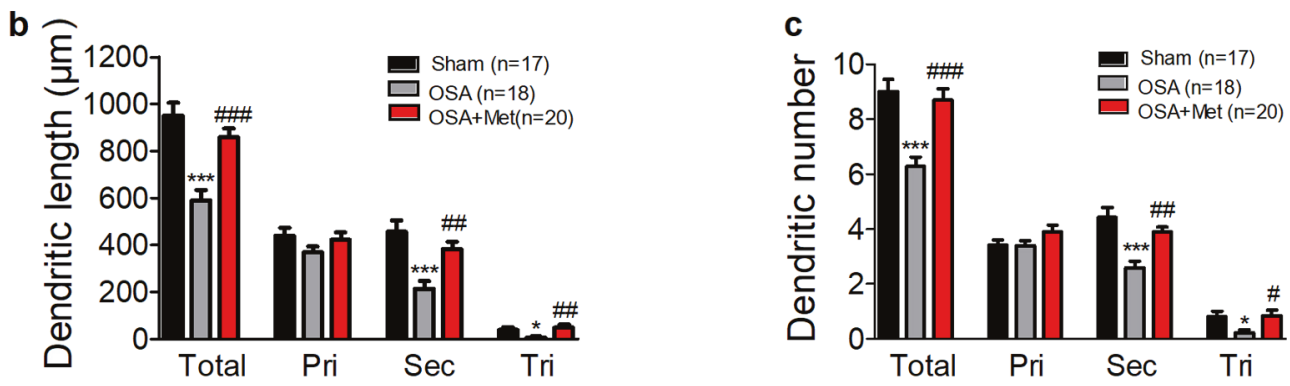

d
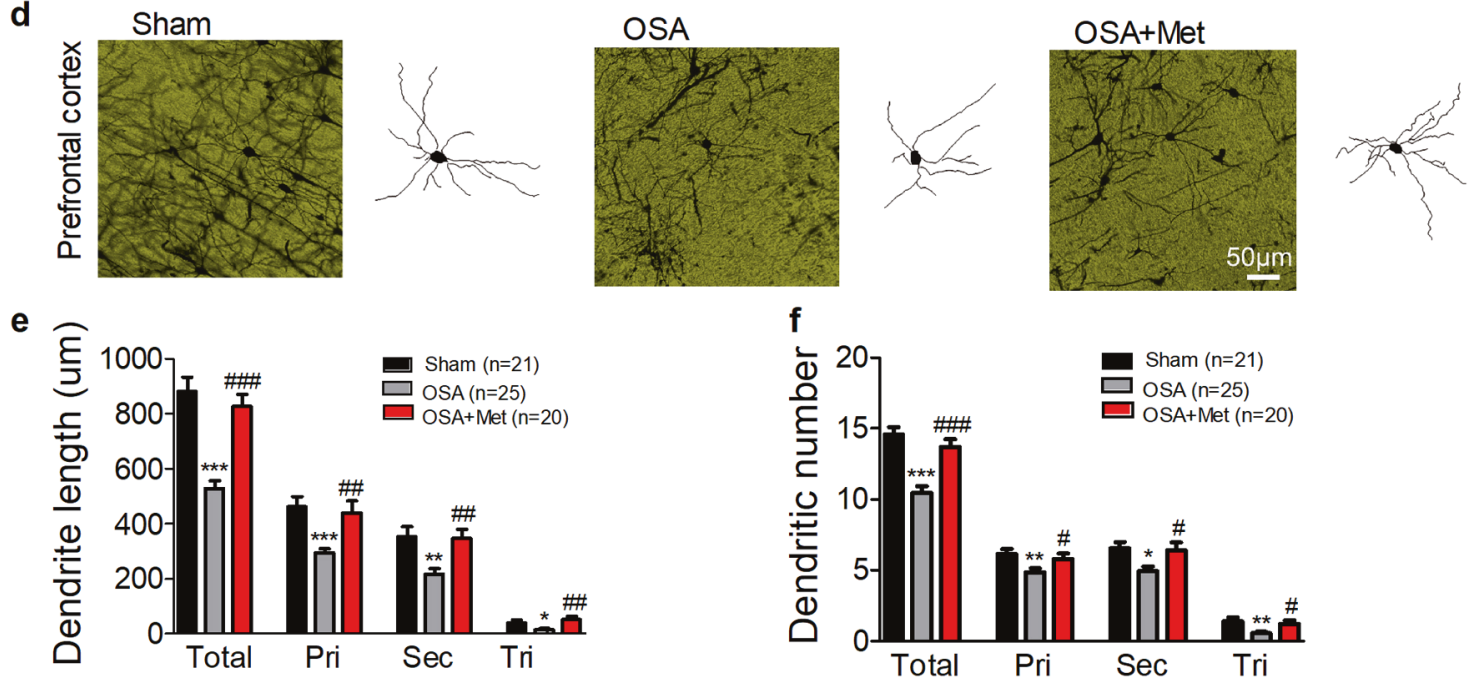

Fig. 4 Metoprolol alleviates granular cell degeneration in the hippocampal DG and nonpyramidal neuron regression in the prefrontal cortex of the OSA model. a Representative photomicrograph (left) and tracing image (right) of granular neurons of the hippocampal DG region from the sham control group (left), OSA group (middle) and OSA treated with metoprolol group (right), scale bar $=50 \mu \mathrm{m}$. b, c Quantification of the dendrite length (b) and dendrite number (c) of hippocampal granular cells. d Representative photomicrograph (left) and tracing image (right) of nonpyramidal neurons in the prefrontal cortex. e, $\mathbf{f}$ Quantification of the dendrite length (e) and dendrite number (f) of prefrontal nonpyramidal cells. ${ }^{*} P<0.05$, ${ }^{* *} P<0.01,{ }^{* * *} P<0.001$ vs. sham control group, ${ }^{\#} P<0.05,{ }^{\# \#} P<0.01$, \#\#\# $P<0.001$ vs. OSA group, values are expressed as the mean \pm SEM.

oxygen concentration could be controlled according to the experimental design [17-19, 50-52]. In addition, controlling the intrathoracic pressure by upper-airway obstruction through placing a plastic cannula around the trachea, and keeping the lower airway open by applying recurrent negative pressure swings to the upper airway through attaching a customized mask to a dual lumen cannula were also used in rodents [17]. As large animals, beagles are also commonly used for OSA research because their metabolic, physiological and anatomical characteristics are closer to those of humans [20]. It has been reported that tracheostomy by clamping a tube onto the trachea was a good method to establish a canine OSA animal model because it could elicit similar electroencephalographic (EEG) and nuchal electromyographic (EMG) characteristics to OSA patients [21, 53]. In this protocol, we could regulate the duration of obstruction and ventilation to control the number of apneas per hour during sleep that could reflect the presence and severity of OSA [3]. Therefore, we chose this method to establish the chronic OSA model in beagles. The time-dependent decrease in negative intra-airway pressure after valve occlusion and the increased HIF-1a expression indicated that we successfully established the beagle model of OSA.

Neuronal circuitry integrity is the structural basis for maintaining normal communication across neurons. The diversity of dendritic structures enables neurons to implement their circuit functions during learning and memory [33]. Spines, as the dendritic protrusions, regulate synaptic plasticity functions in learning and memory consolidation [45]. The alterations in dendrites and spines could be the result of changes in environment, lesions, stress, memory formation or hormone changes during adulthood life [54]. By putting animals into an oxygen-controlled chamber, previous studies reported that the overall hippocampal spine density was dramatically decreased in adult C57 mice [19] and Sprague-Dawley rats following $\mathrm{IH}$ exposure for 1 week [50]. However, no study has focused on large animals, which may reflect that their physiopathological status is close to that of humans. In the present study, by performing tracheostomy, we first reported that OSA for 3 months in beagles 

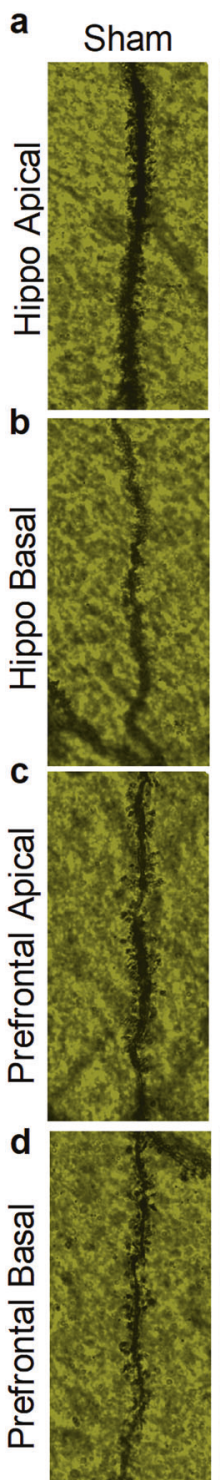
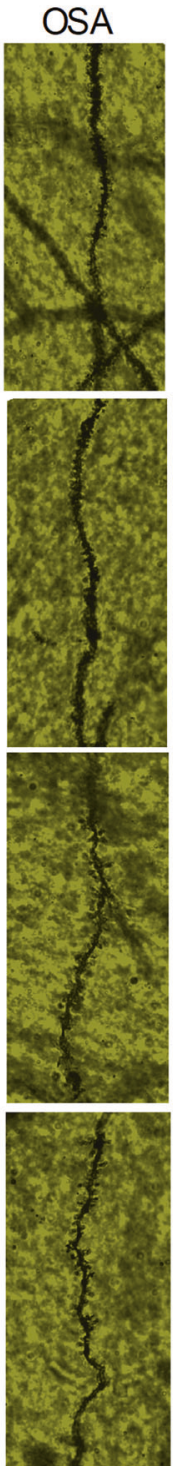
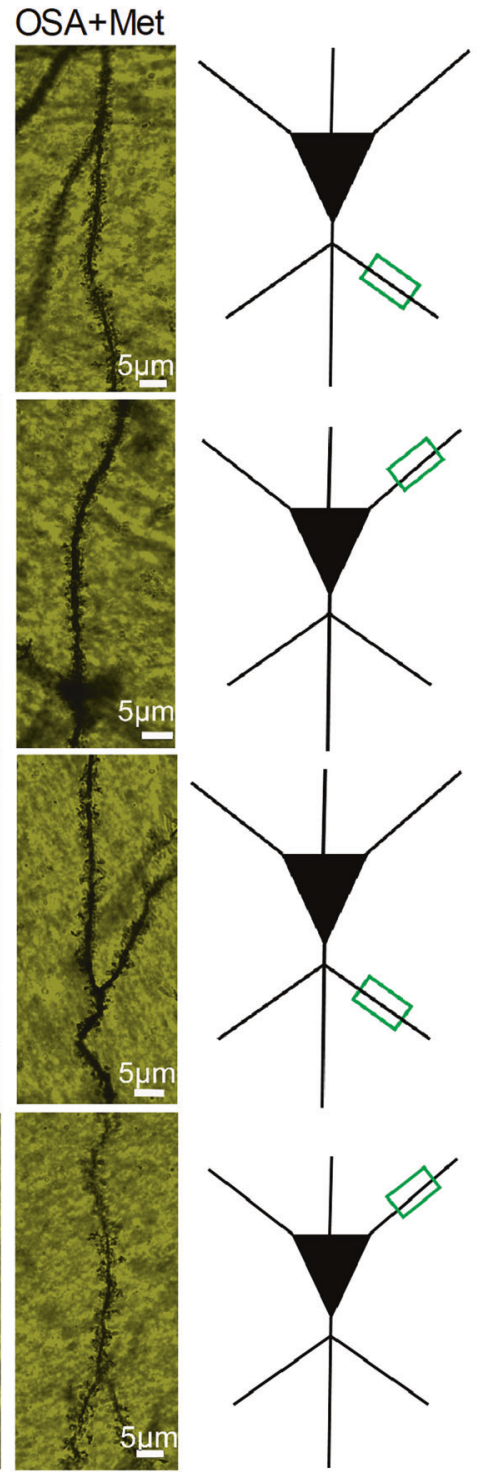

e

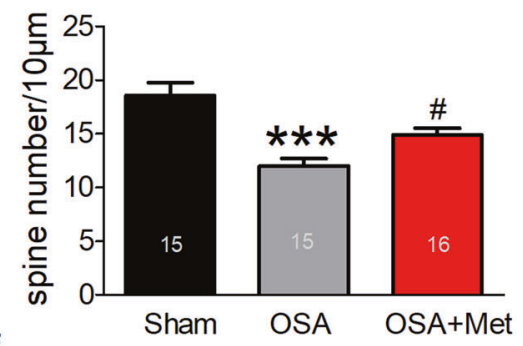

f

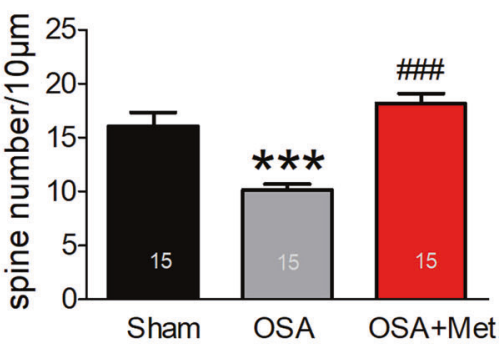

g

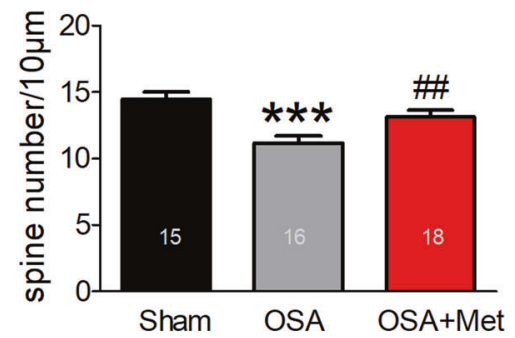

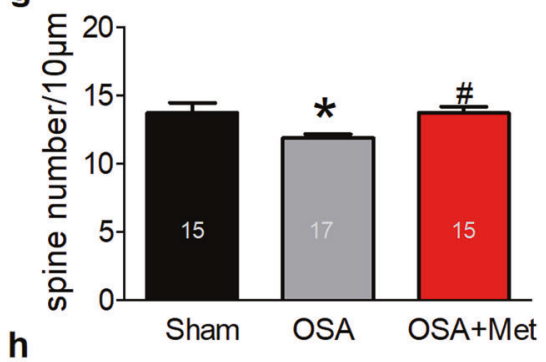

Fig. 5 Metoprolol ameliorates the reduction in dendritic spine density in the OSA canine model. a-d Representative photomicrograph schematic diagram of selected dendritic spines of the apical dendrites (a) and basal dendrites (b) of hippocampal and prefrontal cortical apical dendrites (c) and basal dendrites (d) from the sham control group (left), OSA group (middle) and OSA treated with metoprolol group (right), scale bar $=5 \mu \mathrm{m}$. e, $\mathbf{f}$ Quantification of apical (e) and basal (f) dendritic spine density in hippocampal dendrites. $\mathbf{g}, \mathbf{h}$ Quantification of apical ( $\mathbf{g}$ ) and basal (h) dendritic spine density of prefrontal cortical dendrites. ${ }^{*} P<0.05,{ }^{* * *} P<0.001$ vs. sham control group, $\# P<0.05, \# \# P<0.01, \# \# \#<$ 0.001 vs. OSA group, values are expressed as the mean \pm SEM.

led to the marked morphological remodeling of dendrites in both their hippocampi and prefrontal cortices. In more detail, dendritic architecture consists of primary dendrites arising from the neuron soma and higher order dendrites emerging from primary dendrites [55]. The above study merely quantified the overall apical and basal dendrite length without classifying the dendrites into different orders [50]. In the present study, we found that, for the pyramidal neurons, chronic OSA dramatically decreased the length and number of the total, secondary and tertiary basal dendrites in the hippocampi and prefrontal cortices, as well as in the apical dendrites in the prefrontal cortices. However, chronic OSA did not affect the secondary apical dendrites in the hippocampal CA1 of OSA beagles. Interestingly, the primary dendrites were not changed in any of the pyramidal neurons of the hippocampi and prefrontal cortices. Moreover, although we found that chronic OSA could induce a decrease in the length and number of all primary, secondary and tertiary dendrites of the nonpyramidal cells in the cortices, it affected the total, secondary and tertiary dendrites without changing the primary dendrites of the granular neurons in the hippocampi. The results suggested that the higher order dendrites (secondary and tertiary) were more sensitive to chronic OSA. A previous study found that chronic brain hypoperfusion induced dendritic degeneration, displaying a dying-back process [34]. Here, we provide evidence that chronic OSA may also lead to a dying-back process of dendritic degeneration. Furthermore, we found that the number and length of nonpyramidal neuron dendrites were decreased in all dendrites, which suggested that the prefrontal cortex nonpyramidal neurons may be more vulnerable to OSA. However, the molecular mechanism of this kind of dying-back degeneration of dendrites needs to be explored further.

BDNF plays a positive role in regulating dendrite and spine formation and remodeling in physiological and pathological conditions [56]. Surprisingly, we found that the BDNF level was decreased in the hippocampal CA1 and DG, as well as in the prefrontal cortices of chronic OSA beagles, and that metoprolol 
a
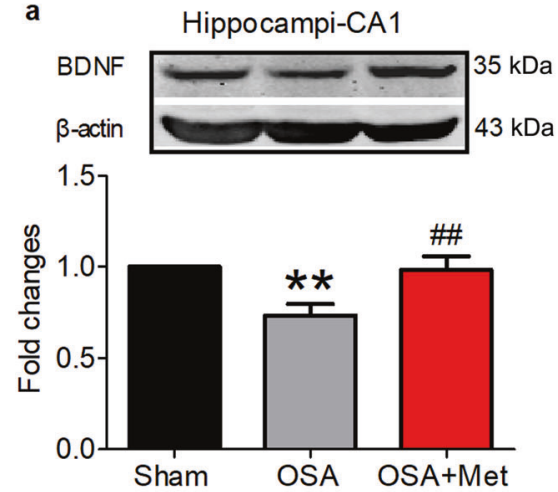

b
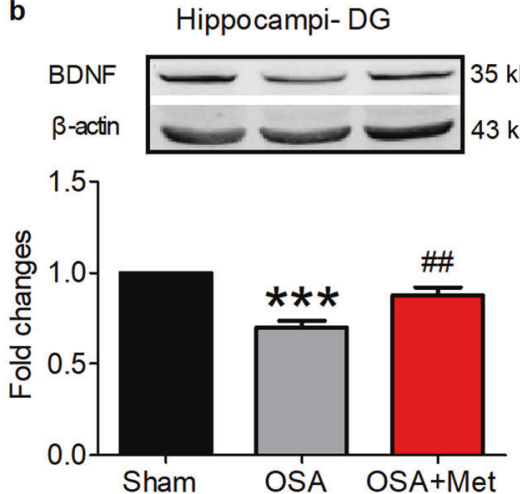

C
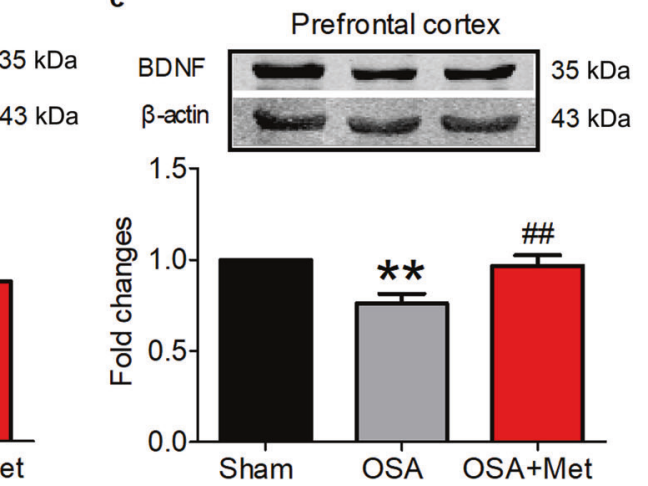

Fig. 6 BDNF level in the hippocampi and prefrontal cortices of sham, OSA and metoprolol-treated OSA beagles. a-c BDNF expression level in the hippocampal CA1 (a) ( $n=4$, with four beagles per group) and hippocampal DG regions (b) ( $n=5$, with four beagles per group) and the prefrontal cortex (c) $(n=5$, with four beagles per group) from the sham group, OSA group and OSA treated with metoprolol group. ${ }^{* *} P<0.01,{ }^{* * *} P<0.001$ vs. sham control group, ${ }^{\# \#} P<0.01$ vs. OSA group, values are expressed as the mean \pm SEM.

administration significantly reversed the decrease in the level of BDNF. This result suggested that BDNF might be involved in the action of metoprolol on dendritic remodeling induced by chronic OSA. However, there is no canine-specific BDNF-siRNA/shRNA on the market, which limits experiments using a gene manipulation strategy to identify whether BDNF is the target of metoprolol. Notably, a previous study showed that hyperbaric oxygen therapy could reduce dendritic/synaptic degeneration via the BDNF/TrkB signaling pathways in spinal cord injury rats [57]. The BDNF downstream signaling pathways, including Akt, PKC, and ERK, were reported to be involved in running-exercise-delayed neurodegeneration of $A D$ (APP/PS1) transgenic mice [58]. We hypothesized that BDNF/TrkB signaling might be involved in the neuroprotection of metoprolol on dendritic remodeling induced by chronic OSA, which needs to be explored further.

There is a close relationship between sleep and the autonomic nervous system. OSA leads to the dysregulation of many biological pathways, the most important of which is the deregulation of the autonomic nervous system [22]. Autonomic nervous system disorders are commonly seen in CNS diseases [24-26], and are considered as potential biomarkers for premotor PD [26]. In early case of $A D$ patients, a loss of dendritic branching and a decrease in dendritic spines were shown in the suprachiasmatic nuclei, the supraoptic nuclei, and the paraventricular nuclei, which were considered to be related to the disability of autonomic functions [59]. However, whether sympathetic or parasympathetic hyperactivation could directly induce dendritic remodeling remains unclear. In a previous study, we found that the chronic OSA canine model showed autonomic nervous system disturbance, including sympathetic and parasympathetic hyperinnervation [23]. Here, we first reported that the autonomic nervous system disability induced by chronic OSA may be involved in neuronal dendrite morphological remodeling. The inhibition of autonomic nerve hyperactivity by metoprolol could prevent cardiac apoptosis, atrial fibrillation and profibrotic remodeling of epicardial adipose tissue in a canine model of chronic OSA [27-29], and could lower blood pressure in hypertensive patients with OSA [38]. Herein, our findings are the first to demonstrate that the inhibition of autonomic nerve hyperactivity by metoprolol could also protect against chronic OSA-induced neuronal dendritic remodeling in the hippocampus and prefrontal cortex.

Taken together, our study demonstrated for the first time that metoprolol could ameliorate dendritic degeneration and spine loss in the hippocampi and prefrontal cortices of beagles following chronic OSA. Our research provided direct evidence that chronic OSA impaired dendritic integrity and spine density. The findings of the present study imply that the autonomic nervous system may participate in chronic OSA-induced neuronal dendritic remodeling, and that inhibiting autonomic nerve hyperactivity by metoprolol may be one of the strategies to prevent neurodegenerative disease with OSA.

\section{ACKNOWLEDGEMENTS}

This work was supported by the National Natural Science Foundation of China (81870849 and 81671052) and the Key project of the Natural Science Foundation of Heilongjiang province (ZD2018004) to J.A.

\section{AUTHOR CONTRIBUTIONS}

$J A$ and $Y L$ designed the study. LY, JZ, YQ, QS, TTL, LMY, JMD, XKL, RYW, YSH and SZ performed experiments and analyzed the data. LY wrote the manuscript. All authors discussed the results.

\section{ADDITIONAL INFORMATION}

Competing interests: The authors declare no competing interests.

\section{REFERENCES}

1. Young T, Skatrud J, Peppard PE. Risk factors for obstructive sleep apnea in adults. JAMA. 2004;291:2013-6.

2. Jordan AS, McSharry DG, Malhotra A. Adult obstructive sleep apnoea. Lancet. 2014;383:736-47.

3. Sarkar P, Mukherjee S, Chai-Coetzer CL, McEvoy RD. The epidemiology of obstructive sleep apnoea and cardiovascular disease. J Thorac Dis. 2018;10: S4189-200.

4. Torres G, Sanchez-de-la-Torre M, Barbe F. Relationship between OSA and hypertension. Chest. 2015;148:824-32.

5. Patel N, Donahue C, Shenoy A, Patel A, El-Sherif N. Obstructive sleep apnea and arrhythmia: a systemic review. Int J Cardiol. 2017;228:967-70.

6. Khattak HK, Hayat F, Pamboukian SV, Hahn HS, Schwartz BP, Stein PK. Obstructive sleep apnea in heart failure: review of prevalence, treatment with continuous positive airway pressure, and prognosis. Tex Heart Inst J. 2018;45:151-61.

7. Glantz H, Thunstrom E, Johansson MC, Wallentin Guron C, Uzel H, et al. Obstructive sleep apnea is independently associated with worse diastolic function in coronary artery disease. Sleep Med. 2015;16:160-7.

8. Ryan CM, Battisti-Charbonney A, Sobczyk O, Mikulis DJ, Duffin J, Fisher JA, et al. Evaluation of cerebrovascular reactivity in subjects with and without obstructive sleep apnea. J Stroke Cerebrovasc Dis. 2018;27:162-8.

9. Gagnon K, Baril AA, Montplaisir J, Carrier J, Chami S, Gauthier S, et al. Detection of mild cognitive impairment in middle-aged and older adults with obstructive sleep apnoea. Eur Respir J. 2018;52:1801137.

10. Liguori C, Mercuri NB, Izzi F, Romigi A, Cordella A, Sancesario G, et al. Obstructive sleep apnea is associated with early but possibly modifiable Alzheimer's disease biomarkers changes. Sleep. 2017;40:zsx011. https://doi.org/10.1093/sleep/zsx011.

11. Quaranta VN, Carratu P, Damiani MF, Dragonieri S, Capozzolo A, Cassano A, et al. The prognostic role of obstructive sleep apnea at the onset of amyotrophic lateral sclerosis. Neurodegener Dis. 2017;17:14-21. 
12. Saunamaki T, Jehkonen M. Depression and anxiety in obstructive sleep apnea syndrome: a review. Acta Neurol Scand. 2007;116:277-88

13. Oyama F, Miyazaki H, Sakamoto N, Becquet C, Machida Y, Kaneko K, et al. Sodium channel beta4 subunit: down-regulation and possible involvement in neuritic degeneration in Huntington's disease transgenic mice. J Neurochem. 2006;98:518-29.

14. Spires-Jones T, Knafo S. Spines, plasticity, and cognition in Alzheimer's model mice. Neural Plast. 2012;2012:319836.

15. Fogarty MJ, Mu EW, Noakes PG, Lavidis NA, Bellingham MC. Marked changes in dendritic structure and spine density precede significant neuronal death in vulnerable cortical pyramidal neuron populations in the SOD1(G93A) mouse model of amyotrophic lateral sclerosis. Acta Neuropathol Commun. 2016;4:77. https:// doi.org/10.1186/s40478-016-0347-y.

16. Csabai D, Wiborg O, Czeh B. Reduced synapse and axon numbers in the prefrontal cortex of rats subjected to a chronic stress model for depression. Front Cell Neurosci. 2018;12:24. https://doi.org/10.3389/fncel.2018.00024.

17. Davis EM, O'Donnell CP. Rodent models of sleep apnea. Respir Physiol Neurobiol. 2013;188:355-61.

18. Kheirandish L, Gozal D, Pequignot JM, Pequignot J, Row BW. Intermittent hypoxia during development induces long-term alterations in spatial working memory, monoamines, and dendritic branching in rat frontal cortex. Pediatr Res. 2005;58:594-9.

19. Xu LH, Xie H, Shi ZH, Du LD, Wing YK, Li AM, et al. Critical role of endoplasmic reticulum stress in chronic intermittent hypoxia-induced deficits in synaptic plasticity and long-term memory. Antioxid Redox Signal. 2015;23:695-710.

20. Toth LA, Bhargava P. Animal models of sleep disorders. Comp Med. 2013;63:91-104

21. Kimoff RJ, Makino H, Horner RL, Kozar LF, Lue F, Slutsky AS, et al. Canine model of obstructive sleep apnea: model description and preliminary application. J Appl Physiol (1985). 1994;76:1810-7.

22. Tobaldini E, Costantino G, Solbiati M, Cogliati C, Kara T, Nobili L, et al. Sleep, sleep deprivation, autonomic nervous system and cardiovascular diseases. Neurosci Biobehav Rev. 2017;74:321-9.

23. Zhao J, Xu W, Yun F, Zhao H, Li W, Gong Y, et al. Chronic obstructive sleep apnea causes atrial remodeling in canines: mechanisms and implications. Basic Res Cardiol. 2014;109:427.

24. Haj Kheder S, Heller J, Bar JK, Wutzler A, Menge BA, Juckel G. Autonomic dysfunction of gastric motility in major depression. J Affect Disord. 2018;226:196-202.

25. Femminella GD, Rengo G, Komici K, lacotucci P, Petraglia L, Pagano G, et al. Autonomic dysfunction in Alzheimer's disease: tools for assessment and review of the literature. J Alzheimers Dis. 2014;42:369-77.

26. Palma JA, Kaufmann H. Autonomic disorders predicting Parkinson's disease. Parkinsonism Relat Disord. 2014;20(Suppl 1):S94-8.

27. Sun L, Yan S, Wang $X$, Zhao S, Li H, Wang $Y$, et al. Metoprolol prevents chronic obstructive sleep apnea-induced atrial fibrillation by inhibiting structural, sympathetic nervous and metabolic remodeling of the atria. Sci Rep. 2017;7:14941.

28. Li W, Yan S, Zhao J, Ding X, Zhang S, Wang D, et al. Metoprolol inhibits cardiac apoptosis and fibrosis in a canine model of chronic obstructive sleep apnea. Cell Physiol Biochem. 2015;36:1131-41.

29. Dai H, Yuan Y, Yin S, Zhang Y, Han Y, Sun L, et al. Metoprolol inhibits profibrotic remodeling of epicardial adipose tissue in a canine model of chronic obstructive sleep apnea. J Am Heart Assoc. 2019;8:e011155.

30. Coenen VA, Gielen FL, Castro-Prado F, Abdel Rahman A, Honey CR. Noradrenergic modulation of subthalamic nucleus activity in human: metoprolol reduces spiking activity in microelectrode recordings during deep brain stimulation surgery for Parkinson's disease. Acta Neurochir (Wien). 2008;150:757-62. discussion 762.

31. Nair $A B$, Jacob $S$. A simple practice guide for dose conversion between animals and human. J Basic Clin Pharm. 2016;7:27-31.

32. Ke Q, Costa M. Hypoxia-inducible factor-1 (HIF-1). Mol Pharmacol. 2006;70:1469-80.

33. Hausser M, Spruston N, Stuart GJ. Diversity and dynamics of dendritic signaling. Science. 2000;290:739-44.

34. Chen X, Jiang XM, Zhao LJ, Sun LL, Yan ML, Tian Y, et al. MicroRNA-195 prevents dendritic degeneration and neuron death in rats following chronic brain hypoperfusion. Cell Death Dis. 2017;8:e2850.
35. Jiang X, Chai GS, Wang ZH, Hu Y, Li XG, Ma ZW, et al. CaMKII-dependent dendrite ramification and spine generation promote spatial training-induced memory improvement in a rat model of sporadic Alzheimer's disease. Neurobiol Aging. 2015;36:867-76.

36. Wu YK, Fujishima K, Kengaku M. Differentiation of apical and basal dendrites in pyramidal cells and granule cells in dissociated hippocampal cultures. PLoS One. 2015;10:e0118482.

37. Linz D, Schotten U, Neuberger HR, Bohm M, Wirth K. Negative tracheal pressure during obstructive respiratory events promotes atrial fibrillation by vagal activation. Heart Rhythm. 2011;8:1436-43.

38. Shi J, Yuan Y, Deng X, Pan Y, He M, Liu G, et al. Metoprolol has a similar therapeutic effect as amlodipine on BP lowering in hypertensive patients with obstructive sleep apnea. Sleep Breath 2019;23:227-33.

39. Alkadhi KA. Cellular and molecular differences between area CA1 and the dentate gyrus of the hippocampus. Mol Neurobiol. 2019;56:6566-80.

40. Kubota $Y$, Karube F, Nomura M, Kawaguchi Y. The diversity of cortical inhibitory synapses. Front Neural Circuits. 2016;10:27. https://doi.org/10.3389/ fncir.2016.00027.

41. Brown SM, Henning S, Wellman CL. Mild, short-term stress alters dendritic morphology in rat medial prefrontal cortex. Cereb Cortex. 2005;15:1714-22.

42. Liston C, Miller MM, Goldwater DS, Radley JJ, Rocher AB, Hof PR, et al. Stressinduced alterations in prefrontal cortical dendritic morphology predict selective impairments in perceptual attentional set-shifting. J Neurosci. 2006;26:7870-4.

43. Radley JJ, Rocher AB, Miller M, Janssen WG, Liston C, Hof PR, et al. Repeated stress induces dendritic spine loss in the rat medial prefrontal cortex. Cereb Cortex. 2006;16:313-20.

44. Li WY, Chang YC, Lee $\sqcup$, Lee LJ. Prenatal infection affects the neuronal architecture and cognitive function in adult mice. Dev Neurosci. 2014;36:359-70.

45. Berry KP, Nedivi E. Spine dynamics: are they all the same? Neuron. 2017;96:43-55.

46. Leal G, Afonso PM, Salazar IL, Duarte CB. Regulation of hippocampal synaptic plasticity by BDNF. Brain Res. 2015;1621:82-101.

47. Chan JP, Cordeira J, Calderon GA, lyer LK, Rios M. Depletion of central BDNF in mice impedes terminal differentiation of new granule neurons in the adult hippocampus. Mol Cell Neurosci. 2008;39:372-83.

48. Miranda M, Morici JF, Zanoni MB, Bekinschtein $\mathrm{P}$. Brain-derived neurotrophic factor: a key molecule for memory in the healthy and the pathological brain. Front Cell Neurosci. 2019;13:363.

49. Sidorova YA, Volcho KP, Salakhutdinov NF. Neuroregeneration in Parkinson's disease: from proteins to small molecules. Curr Neuropharmacol. 2019;17:268-87.

50. Lam CS, Li JJ, Tipoe GL, Youdim MBH, Fung ML. Monoamine oxidase A upregulated by chronic intermittent hypoxia activates indoleamine 2,3-dioxygenase and neurodegeneration. PLoS One. 2017;12:e0177940.

51. Raman L, Hamilton KL, Gewirtz JC, Rao R. Effects of chronic hypoxia in developing rats on dendritic morphology of the CA1 subarea of the hippocampus and on fear-potentiated startle. Brain Res. 2008;1190:167-74.

52. Wallace MG, Hartle KD, Snow WM, Ward NL, Ivanco TL. Effect of hypoxia on the morphology of mouse striatal neurons. Neuroscience. 2007;147:90-6.

53. Yu L, Li X, Huang B, Zhou X, Wang M, Zhou L, et al. Atrial fibrillation in acute obstructive sleep apnea: autonomic nervous mechanism and modulation. J Am Heart Assoc. 2017;6:e006264.

54. Tavosanis G. Dendritic structural plasticity. Dev Neurobiol. 2012;72:73-86.

55. Charych El, Akum BF, Goldberg JS, Jornsten RJ, Rongo C, Zheng JQ, et al. Activityindependent regulation of dendrite patterning by postsynaptic density protein PSD-95. J Neurosci. 2006;26:10164-76.

56. von Bohlen Und Halbach O, von Bohlen Und Halbach V. BDNF effects on dendritic spine morphology and hippocampal function. Cell Tissue Res. 2018;373:729-41.

57. Ying $X$, Tu W, Li S, Wu Q, Chen X, Zhou Y, et al. Hyperbaric oxygen therapy reduces apoptosis and dendritic/synaptic degeneration via the BDNF/TrkB signaling pathways in SCI rats. Life Sci. 2019;229:187-99.

58. Lin TW, Shih $\mathrm{YH}$, Chen SJ, Lien $\mathrm{CH}$, Chang $\mathrm{CY}$, Huang TY, et al. Running exercise delays neurodegeneration in amygdala and hippocampus of Alzheimer's disease (APP/PS1) transgenic mice. Neurobiol Learn Mem. 2015;118:189-97.

59. Baloyannis SJ, Mavroudis I, Mitilineos D, Baloyannis IS, Costa VG. The hypothalamus in Alzheimer's disease: a Golgi and electron microscope study. Am J Alzheimers Dis Other Demen. 2015;30:478-87. 